\title{
Aquatic animal telemetry: A panoramic window into the underwater world
}

\author{
Nigel E. Hussey ${ }^{1}$, \\ ${ }^{1}$ University of Windsor - Biological Sciences, 401 Sunset Avenue, Windsor, Ontario. \\ N9B 3P4. Canada.
}

BACKGROUND: Global aquatic environments are changing profoundly as a result of human actions; consequently, so too are the ways in which organisms are distributing themselves through space and time. Our ability to predict organism and community responses to these alterations will be dependent on knowledge of animal movements, interactions, and how the physiological and environmental processes underlying them shape species distributions. These patterns and processes ultimately structure aquatic ecosystems and provide the wealth of ecosystem services upon which humans depend. Until recently, the vast size, opacity, and dynamic nature of the aquatic realm have impeded our efforts to understand these ecosystems. With rapid technological advancement over the past several decades, a suite of electronic tracking devices (e.g., acoustic and satellite transmitters) that can remotely monitor animals in these challenging environments are now available. Aquatic telemetry technology is rapidly accelerating our ability to observe animal behavior and distribution and, as a consequence, is fundamentally altering our understanding of the structure and function of global aquatic ecosystems. These advances provide the toolbox to define how future global aquatic management practices must evolve.

ADVANCES: Aquatic telemetry has emerged through technological advances in miniaturization, battery engineering, and software and hardware development, allowing the monitoring of organisms whose habitats range from the poles to the tropics and the photic zone to the abyssal depths. This is enabling the characterization of the horizontal and vertical movements of individuals, populations, and entire communities over scales of meters to tens of thousands of kilometers and over time frames of hours to years and even over the entire lifetimes of individuals. Electronic tags can now be equipped with sensors that measure ambient physical parameters (depth, temperature, conductivity, fluorescence), providing simultaneous monitoring of animals' environments. By linking telemetry with biologgers (e.g., jaw-motion sensors), it is possible to monitor individual feeding events. In addition, other devices on instrumented animals can communicate with one another, providing insights into predator-prey interactions and social behavior. Coupling telemetry with minute nonlethal biopsy allows understanding of how trophic dynamics, population connectivity, and gene-level basis for organismal health and condition relate to movement. These advances are revolutionizing the scope and scales of questions that can be addressed on the causes and consequences of animal distribution and movement.

OUTLOOK: Aquatic animal telemetry has advanced rapidly, yet new challenges present themselves in coordination of monitoring across large-spatial scales (ocean basins), data sharing, and data assimilation. The continued advancement of aquatic telemetry lies in establishing and maintaining accessible and cost-effective infrastructure and in promoting 
multidisciplinary tagging approaches to maximize cost benefits. A united global network and centralized database will provide the mechanism for global telemetry data and will promote a transparent environment for data sharing that will, in turn, increase global communication, scope for collaboration, intellectual advancement, and funding opportunities. An overarching global network will realize the potential of telemetry, which is essential for advancing scientific knowledge and effectively managing globally shared aquatic resources and their ecosystems in the face of mounting human pressures and environmental change. 